\section{Michael Hölzer, Wolfgang Wöller, Götz Berberich (Hrsg.): Stationäre Psychotherapie. Von der Anmeldung bis zur Entlassung}

\author{
Schattauer, 2018, Stuttgart, 640 Seiten, 82,00€(D)
}

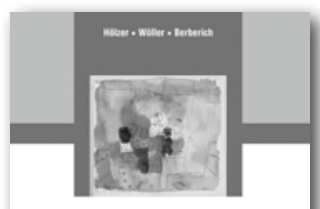

Stationäre Psychotherapie
Sisions tationäre Psychotherapie hat in Deutschand wie in keinem anderen Land Europas eine lange Tradition und ist ein fester Teil psychosomatischer/psychotherapeutischer Versorgung von PatientInnen geworden. Nun legen Michael Hölzer und seine Kollegen, alles langjährig praktizierende Chefärzte, ein modernes, umfangreiches Werk zur stationären Psychotherapie vor. Die Inhalte (38 Kapitel beginnend von der Anmeldung bis zur Entlassung) und der Umfang des Buches (627 Seiten) zeigen dem / der Leserln schon bei kurzer Durchsicht, mit welcher komplexen Thematik sich stationäre Psychotherapie heutzutage auseinandersetzen muss. Kritische Themen wie Beschleunigung und damit immer kürzer werdende Behandlungszeiten, ambulante Behandlung versus stationäre Behandlung, Entwicklungs- und Gesundungsräume für weniger privilegierte Schichten und die damit verbundene Regressionsgefahr, die Gefahr, als Reparaturanstalten für einen problematischen Kapitalismus zu fungieren, in dem Menschen unter schwierigsten Arbeitsbedingungen ganz einfach krank werden, würde man in einem solchen Buch nicht erwarten. Zumeist glänzen vergleichbare Werke mit einer Theorie- und Methodenselbstverliebtheit, ohne auch die gesellschaftlichen Bedingungen seelischer Erkrankung zu reflektieren. Gewiss, auch die Autoren dieses Buches konzentrieren sich auf das „solide Handwerk“, das eine gute stationäre psychotherapeutische Arbeit ausmacht, und beziehen sehr wertschätzend das gesamte Setting von der Bedeutung der Teamarbeit, der Pflege, über die Spezialtherapien bis hin zur Bedeutung des therapeutischen Milieus ein. Und damit wären wir beim Thema.
Ich will mich in dieser Rezension auf die Ausführungen zu den Spezialtherapien fokussieren, denn das wird den / die Leserln wohl am meisten interessieren.

In sechs Kapiteln nehmen die Autoren dabei direkt oder im weiteren Sinne Bezug. Körperorientierte Therapien werden beschrieben, die Kunst- und Gestaltungstherapie, Musiktherapie und sporttherapeutische Anwendungen. Wir wissen, dass sich stationäre Einrichtungen erheblich im Einsatz spezialtherapeutischer Versorgung unterscheiden. Dennoch ist eine stationäre Versorgung heute ohne die Konzentrative Bewegungstherapie, die Integrative Bewegungstherapie, die Tanztherapie und sporttherapeutische Anwendungen mit mehr funktional-motorischer Ausrichtung kaum mehr denkbar. Der ursprüngliche Paradigmenwechsel in der stationären Therapie von vorwiegend psychoanalytisch-psychodynamischen Ansätzen hin zu mehr verhaltenstherapeutischen und systemischen Verfahrensweisen hat auch vor den Spezialtherapien keinen Halt gemacht und wird bereits im Einleitungskapitel (S.12) erwähnt.

Im Kapitel 14 „Bewegen und Wahrnehmen Körperorientierte Therapien“ beschreiben Hölzer und Heck die große und heterogene Gruppe der körperorientierten Therapien von den Quellen bis zur modernen Bedeutung des Embodiments und fokussieren sich schließlich auf die Konzentrative Bewegungstherapie (KBT) und die Integrative Bewegungstherapie (IBT). Sie schaffen dann noch eine Verbindung zu den „Verwandten aus Fernost“: Qigong, Tai-Chi und Yoga. Und es folgt wiederum eine Rückverbindung zur progressiven Muskelrelaxation, zum 
Autogenen Training und zum Biofeedback. Natürlich wird der/die ein oder andere Leserln enttäuscht sein, das eigene Verfahren nicht in diesem Buch zu finden. Den Autoren muss jedoch beschieden werden, dass sie sich damit auf zwei Verfahren konzentriert haben, die sich bereits seit den 1960er und 1970er Jahren im klinischen Setting finden und damit auf eine über 50-jährige klinische Praxis zurückblicken können. Östliche Verfahren scheinen sich heute in der klinisch-übungszentrierten Arbeit von Entspannung und Stressregulierung ebenso (oder, wie die Autoren meinen, „mehr als nur Entspannung“) einen festen Platz erkämpft zu haben. Die Arbeiten von Kabat-Zinn haben hier sicherlich einen wichtigen Beitrag zur Akzeptanz geleistet.

Sportliche Aktivitäten und Sporttherapie werden in einem eigenen Kapitel (18) gewürdigt. Die wissenschaftliche Datenlage bei Angststörungen, depressiven Störungen, somatoformen Störungen und weiteren Krankheitsbildern ist inzwischen so überzeugend, dass körperliche Aktivität gerade auch im Hinblick auf die Motivationsbildung beim Patienten heute in einer psychosomatisch-psychotherapeutischen Klinik kaum mehr wegzudenken ist. Und was mich dann doch noch überrascht hat: dass selbst die Bedeutung des Naturerlebens (S. 235) aufgegriffen wird.

Dies macht das Buch zu etwas Besonderem: Es beschreibt alle zentralen Inhalte einer stationären Behandlung, die Autoren reduzieren sich an einigen Stellen auf das Wesentliche, weisen jedoch auch auf zukünftige Entwicklungen und Herausforderungen hin. Als ich 1994 das Buch von Peter Fürstenau „Entwicklungsförderung durch Therapie“ gelesen habe, faszinierte mich darin besonders der Beitrag zur stationären Psychotherapie im Jahr 2000 (S.163). Fürstenau war ein Visionär. Hölzer und seine Kollegen sind im Schlussbeitrag „Quo vadis?“ (S. 589) vorsichtiger und kritisch, was die Zukunft der stationären Behandlung betrifft, denn die therapeutische, institutionelle und gesamtgesellschaftliche Komplexität hat seitdem immens zugenommen.

Für ambulante SpezialtherapeutInnen, die sich ein Bild über die Komplexität klinischer Behandlungen verschaffen wollen, kann ich dieses Buch uneingeschränkt empfehlen. Für klinisch tätige SpezialtherapeutInnen ist das Werk ein „Muss“.

Martin J. Waibel

DOI 10.2378 / ktb2020.art15d 